\title{
Dificuldade funcional em idosos brasileiros: um estudo com base na Pesquisa Nacional de Saúde (PNS - 2013)
}

\author{
Functional difficulty among elderly Brazilians: a study based on the \\ National Health Survey (PNS - 2013)
}

Camila Zanesco (https://orcid.org/0000-0001-8326-0390) ${ }^{1}$

Danielle Bordin (https://orcid.org/0000-0001-7861-0384) ${ }^{1}$

Celso Bilynkievycz dos Santos (https://orcid.org/0000-0003-2107-8299) ${ }^{1}$

Cristina Berger Fadel (https://orcid.org/0000-0002-7303-5429) ${ }^{1}$

${ }^{1}$ Setor de Ciências

Biológicas e da Saúde,

Universidade Estadual de

Ponta Grossa. Praça Santos

Andrade s/n, Centro.

84010-790 Ponta Grossa

PR Brasil.camila_zanesco@

hotmail.com

\begin{abstract}
The objective of this study was to study the factors that present a high degree of association with functional difficulties among the elderly. A cross-sectional, quantitative study was conducted among individuals aged 60 years or older $(n=$ 23,815). Two dependent variables were considered: Basic Activity of Daily Living and Instrumental Activity of Daily Living, and 42 independent variables related to sociodemographic issues, general health, oral health and the use of health services. For the analysis, the Waikato Environment for Knowledge Analysis software was used. Data set balancing and the dimensionality reduction test were performed through 10-fold cross-validation and lastly, the variables related to the dependent variables were evaluated through logistic regression. Eight variables had a strong association with functional difficulties among the elderly, related to physical aspects, intrinsic factors, social relations, literacy and the use of health services. The results obtained enable the bolstering of planning and execution of strategies in public health, directed to the elderly population, avoiding or minimizing situations of functional difficulty.
\end{abstract}

Key words Interdisciplinary practices, Elderly people, Functional difficulty, Indicators of services
Resumo Objetivou-se, por meio do estudo da Pesquisa Nacional de Saúde, conhecer os fatores que apresentam alto grau de associação com quadros de dificuldade funcional em idosos. Estudo transversal, quantitativo, a amostra constituiu-se das respostas dos indivíduos com 60 anos ou mais $(n=23.815)$. Foram consideradas duas variáveis dependentes, sendo: Atividade Básica de Vida Diária e Atividade Instrumental de Vida Diária, e 42 variáveis independentes, relacionadas a questões sociodemográficas, saúde geral, saúde bucal e uso de serviços de saúde. Para a análise, utilizou-se o software Waikato Environment for Knowledge Analysis. Realizou-se o balanceamento do conjunto de dados, teste de redução de dimensionalidade, por meio da validação cruzada de 10 Fold, e ao final, as variáveis relacionadas com as variáveis dependentes foram avaliadas através da regressão logística. Oito variáveis apresentaram forte associação com quadros de dificuldade funcional em idosos, relacionadas a aspectos físicos, fatores intrínsecos, relações sociais, alfabetização e uso de serviços de saúde. Os resultados alcançados permitem o fortalecimento do planejamento e da execução de estratégias em saúde pública, direcionadas à população idosa, evitando ou minimizando situações de dificuldade funcional.

Palavras-chave Práticas interdisciplinares, Idosos, Dificuldade funcional, Indicadores de serviços 


\section{Introdução}

A elevação no número de indivíduos idosos, somada à transição no perfil epidemiológico da população, vem contribuindo para o aumento da demanda no âmbito dos serviços de saúde públicos e privados ${ }^{1-4}$, além de onerar custos financeiros em nível familiar, comunitário e de socieda$\mathrm{de}^{5}$. Tais mudanças atingem de forma divergente países em situações econômicas heterogêneas, sendo mais recente e intensa naqueles que se encontram em desenvolvimento ${ }^{4}$.

A exposição a situações de risco, doenças e outros agravos ao longo da vida destes indivíduos, somadas à escassez de ações de prevenção contínuas, colaboram para acelerar o processo fisiológico de envelhecimento ${ }^{1,3-5}$. O entrelaçado de acontecimentos apontados culmina nas dificuldades atuais enfrentadas pela sociedade em geral, relacionadas ao público idoso ${ }^{1,3,5}$.

O envelhecimento com qualidade representa um desafio atual, sendo que a exiguidade de doenças não mais confere um parâmetro exclusivo de proteção à saúde. Aspectos funcionais considerando capacidades psicocognitivas e físicas são forte indicador de saúde entre indivíduos idosos $^{6}$. Desta forma, compreende-se a direta ligação entre idosos e quadros de dificuldade funcional (DF), entendida como a incapacidade de realização de tarefas pertinentes à vida diária, processo dinâmico e progressivo, multidimensional, fortemente influenciado por fatores genéticos e por condições experimentadas ao longo da vida $^{1,3,4,7,8}$. A DF é subdividida em dois domínios: atividades básicas de vida diária (ABVD), o qual abarca questões relacionadas ao autocuidado, e atividades instrumentais de vida diária (AIVD), que envolve a independência do idoso na comunidade ${ }^{1,3,7,9}$.

O número de idosos com DF tem aumentado significativamente, entretanto, os estudos, em sua maioria, reportam unicamente taxas de prevalência, eximindo-se da investigação de situações ou de condições que potencializam as chances de tais limitações, e quando o fazem consideram grupos populacionais limitados ou causas específicas $^{3,8}$. Neste sentido, o presente estudo assume a provocação de uma investigação ampla, inédita e inadiável sobre o conhecimento do processo do envelhecimento humano e de suas relações.

O nexo existente entre a longevidade e as DF requer a frequente avaliação deste parâmetro, permitindo a construção de bases de conhecimento para a sustentação do planejamento de ações e de intervenções focadas em demandas es- pecíficas $^{10-12}$. Usufruindo da significância e da relevância conquistadas pelos inquéritos de saúde nos últimos anos ${ }^{13-15}$, objetivou-se, conhecer os fatores com alto grau de associação com quadros de dificuldade funcional em idosos brasileiros, considerando condições sociodemográficas, de saúde geral, de saúde bucal e de utilização de serviços de saúde, por meio do estudo da Pesquisa Nacional de Saúde - 2013 (PNS - 2013).

\section{Metodologia}

É um estudo exploratório, transversal e quantitativo, com fonte de dados secundários provenientes da PNS-2013, proposto pelo Ministério da Saúde e dirigido, em 2013, pelo Instituto Brasileiro de Geografia e Estatística (IBGE) ${ }^{16}$. A PNS-2013 foi aprovada pela Comissão Nacional de Ética em Pesquisa para Seres Humanos, do Ministério da Saúde ${ }^{15}$.

A PNS-2013 contou com uma amostra de 205.546 adultos entrevistados em 60.202 domicílios, conduzida por três formulários para coleta de dados, referentes: ao domicílio, aos moradores e ao indivíduo. No presente estudo foram utilizadas apenas as informações dos indivíduos com idade igual ou superior a 60 anos $(n=23.815)$, provenientes dos dois últimos formulários ${ }^{13,14,16}$. A explicação minuciosa em relação ao processo de amostragem e ponderação, bem como sobre o método para coleta de dados, estão disponíveis no relatório da $\mathrm{PNS}^{16}$.

As duas variáveis desfecho, nomeadas de ABVD e AIVD, resultaram da reunião de sete e cinco questões consecutivamente. A variável ABVD resultou das questões: Em geral, que grau de dificuldade tem para comer sozinho(a) com um prato colocado à sua frente, incluindo segurar um garfo, cortar alimentos e beber em um copo?, Em geral, que grau de dificuldade tem para tomar banho sozinho(a) incluindo entrar e sair do chuveiro ou banheira?, Em geral, que grau de dificuldade tem para ir ao banheiro sozinho(a) incluindo sentar e levantar do vaso sanitário?, Em geral, que grau de dificuldade tem para se vestir sozinho(a), incluindo calçar meias e sapatos, fechar o ziper, e fechar e abrir botões?, Em geral, que grau de dificuldade tem para andar em casa sozinho(a) de um cômodo a outro da casa, em um mesmo andar, como do quarto para a sala e cozinha?, Em geral, que grau de dificuldade tem para deitar ou levantar da cama sozinho(a)?, Em geral, que grau de dificuldade tem para sentar ou levantar da cadeira sozinho?. A variável AIVD é resultante das questões: Em geral, 
que grau de dificuldade tem para fazer compras sozinho(a), por exemplo de alimentos, roupas ou medicamentos?, Em geral, que grau de dificuldade tem para administrar as finanças sozinho(a) (cuidar do seu próprio dinheiro)?, Em geral, que grau de dificuldade tem para tomar os remédios sozinho(a)?, Em geral, que grau de dificuldade tem para ir ao médico sozinho(a)?, Em geral, que grau de dificuldade tem para sair sozinho(a) utilizando um transporte como ônibus, metrô, táxi, carro, etc.?.

Todas as questões utilizadas contemplavam as seguintes opções de resposta: 1. Não consegue, 2 . Tem grande dificuldade, 3 . Tem pequena dificuldade, 4 . Não tem dificuldade. Para realizar a análise dos dados a serem utilizados no presente estudo, as opções de resposta foram dicotomizadas em: com dificuldade (1. Não consegue, 2. Tem grande dificuldade, 3 . Tem pequena dificuldade) e sem dificuldade (4. Não tem dificuldade) $)^{2,17}$.

A amostra utilizada no grupo de indivíduos com dificuldade funcional para as atividades básicas e as instrumentais foi composta apenas por indivíduos que apresentaram dificuldade independente do grau para todas as atividades investigadas $^{7,17,18}$. O indivíduo que apresentou limitação em AIVD e ABVD foi incluído no grupo de dificuldade para ABVD. Na sessão de resultados, são apresentadas as frequências absolutas e as relativas da presença de dificuldade em cada variável formadora das variáveis dependentes.

As questões mencionadas foram respondidas em todos os integrantes do domicílio por um único morador, podendo, deste modo, não ter sido respondida pelo idoso. Para o presente estudo foram elencadas questões de interesse para compor as variáveis independentes, relacionadas à: características sociodemográficas (nove variáveis); limitações funcionais e adoecimento (sete variáveis); utilização de serviços de saúde (onze variáveis); internações e urgências médicas (oito variáveis); e condição de saúde bucal (sete variáveis). Nesta etapa todas as variáveis foram tratadas, as numéricas alteradas para categóricas, algumas recategorizadas, enquanto outras dicotomizadas em consonância com o exposto na literatura ${ }^{17}$. Na sequência são expostas as variáveis independentes, consideradas no estudo, e as categorias formadas.

- Características sociodemográficas: sexo (feminino e masculino); idade ( 60 a 64 anos, 65 a 69 anos, 70 a 74 anos, 75 a79 anos, 80 a 84 anos, 86 a 89 anos, 90 a 94 anos, 95 a 99 anos e 100 ou mais anos); cor da pele (branca, preta, amarela, parda, indígena e não respondeu); convivência com o cônjuge (sim e não); estado civil [casado (a), se- parado(a)/divorciado(a), viúvo(a) e solteiro(a)]; alfabetizado (a) (sim e não); nível de educação (não respondeu, alfabetização, nível fundamental, nível médio, graduação ou superior); renda (até 680 Reais, $681>1320$ reais, $1321>2640$ reais, mais de 2641 reais e não informou); região de residência (Norte, Nordeste, Centro-Oeste, Sudeste e Sul).

- Limitações e adoecimento: diagnóstico de alguma doença crônica, física ou mental (sim e não); percepção da condição de saúde geral (boa e ruim); presença de alguma doença crônica, física ou mental que limita de alguma forma suas atividades habituais (sim e não); deixou de realizar quaisquer de suas atividades habituais por motivo de saúde ( deixou de realizar suas atividades habituais por motivo de saúde (1-3 dias, 4-7 dias, 8-11 dias, 12-14 dias e 15 ou mais dias); esteve acamado nas duas últimas semanas (sim e não); número de dias acamado nas duas últimas semanas (1-3 dias, 4-7 dias, 8-11 dias, 12-14 dias e 15 ou mais dias).

- Utilização de serviços de saúde: onde costuma procurar atendimento quanto está doente (farmácia, unidade básica de saúde, centro de especialidades/policlínica pública ou posto de assistência médica/unidade de pronto atendimento, consultório privado, solicitou atendimento no domicílio e outro); costuma procurar o mesmo serviço de saúde sempre (sim e não); já consultou um médico ao longo da vida (já consultou um médico e nunca consultou um médico); número de consultas nos 12 últimos meses (1-3 consultas, 4-6 consultas, 7-9 consultas, 10-14, consultas, 15-19 consultas, 20-29 consultas, 30 ou mais consultas); procurou por algum lugar, serviço ou profissional de saúde para atendimento relacionado à própria saúde nas últimas duas semanas ( $\operatorname{sim}$ e não); motivo pela procura de atendimento (acidente ou lesão, doença, problema odontológico, reabilitação ou terapia, continuação de tratamento, exame complementar de diagnóstico, vacinação, outro atendimento preventivo, solicitação de atestado de saúde e outro); local onde procurou atendimento nas duas últimas semanas (farmácia, unidade básica de saúde, centro de especialidades/policlínica pública/posto de assistência médica/unidade de pronto atendimento, consultório privado, solicitou atendimento no domicílio e outro); teve medicamentos receitados na última consulta ( o principal atendimento de saúde que recebeu no último atendimento (duas últimas semanas) (consulta médica, consulta odontológica, consul- 
ta com outro profissional de saúde, atendimento com agente comunitário de saúde, atendimento na farmácia, vacinação, injeção/curativo/medição de pressão arterial, quimioterapia/radioterapia/hemodiálise/hemoterapia, exames laboratoriais/de imagem/exames complementares de diagnóstico, gesso/imobilização, pequena cirurgia ambulatorial, internação hospitalar, marcação de consulta, práticas complementares como acupuntura/homeopatia/fitoterapia, outro atendimento e não respondeu); o serviço de saúde em que procurou atendimento nas duas últimas semanas era (público, privado, não sabe e não respondeu); utilizou de alguma prática integrativa e complementar (sim e não).

- Internações e urgências médicas: precisou de internação hospitalar (por 24 horas ou mais) nos últimos 12 messes (sim e não); número de internações nos últimos 12 meses (1-3 internações, 4-6 internações, 7-9 internações, 10-14 internações, 15-19 internações, 20-29 internações, 30 ou mais internações); motivo pelo qual precisou de internação hospitalar (tratamento clínico, tratamento psiquiátrico, cirurgia, exames complementares de diagnóstico e outro); tempo que permaneceu internado (a) na última vez (últimos 12 meses) (1-3 dias, 4-6 dias, 7-9 dias, 10-14 dias, 15-19 dias, 2029 dias e 30 ou mais dias); na última vez que foi internado como classifica o atendimento recebido (bom e ruim); nos últimos 12 meses, precisou de atendimento de emergência no domicílio (sim e não); na última vez que teve atendimento de urgência no domicílio, como foi o atendimento recebido (bom e ruim); no último atendimento de emergência no domicílio precisou ser transportado por ambulância (

- Saúde bucal: autopercepção de saúde bucal (boa e ruim); tem dificuldade para se alimentar devido a problemas com os dentes ou dentadura (tem dificuldade, não tem nenhuma dificuldade e não respondeu); perdeu dentes na arcada inferior (não, sim, perdi todos, não se aplica e não respondeu); perdeu dentes na arcada superior (não, sim, perdi todos, não se aplica e não respondeu); número de dentes naturais presentes (menos de 10 dentes, mais de 10 dentes); faz uso de prótese dental (não, próteses dentárias totais em uma das arcadas dentárias/ou para substituir determinado número de dentes, próteses dentárias totais para ambas as arcadas); consultou um cirurgião dentista alguma vez na vida (

Em sequência, a fase de tratamento do banco de dados foi realizado com o balanceamento de todo o conjunto, objetivando equilibrar as classes da variável dependente e favorecendo o processo de análise. Foram testados todos os algoritmos de balanceamento, sendo o Resample o eleito, visto que apresentou melhor eficiência. O Resample consiste em uma ferramenta para pré-processamento de dados, incorporada ao software de mineração de dados Waikato Environment for Knowledge Analysis (WEKA) ${ }^{19}$, podendo ser utilizada em outros softwares que possibilitem a inclusão de algoritmos de mineração de dados. Após, foi realizado o teste de redução de dimensionalidade, usando o método de seleção de atributos e empregando a abordagem filtro oportunizada no WEKA (https://www.cs.waikato.ac.nz/ $\mathrm{ml} /$ weka/index.html). A adequação do software WEKA ao estudo, seu caráter livre e gratuito, corriqueiramente utilizado por profissionais de saúde, devido à eficácia, rapidez e confiabilidade, conduziram a respectiva escolha do mesmo para uso no estudo em questão ${ }^{20}$.

$\mathrm{Na}$ abordagem operou-se com o algoritmo Correlation-based Feature Selection $(\mathrm{CFS})^{19}$, por meio do método de validação cruzada de 10 Fold, sendo essa a opção que apresentou maior compatibilidade com o presente estudo. Fold corresponde ao poder explicativo da variável em porcentagem conforme apresentado posteriormente nos resultados. O teste aponta quais são as variáveis independentes que têm elevada relação com as variáveis dependentes e baixa entre si, excluindo relações de confundimento, permitindo reconhecer as relações verdadeiras com precisão, trabalhando simultaneamente com todas as variáveis elencadas no estudo ${ }^{19}$.

A validação cruzada segmenta os dados em subconjuntos, denominados folds, para a aplicação de um algoritmo de indução de conhecimento. Cada um destes folds será usado como conjunto de testes uma vez. Este processo permite, ao final, o cálculo através da média de resultados obtidos em cada etapa onerando a qualidade do conhecimento gerador ${ }^{19}$.

No modelo final foram apreciadas somente as variáveis que apresentaram grande potencialidade para aclarar a variável desfecho, permitindo autenticar as ligações puras e estritas com as variáveis independentes. Os modelos oriundos do uso desta metodologia são mais fidedignos e explicativos, facultando o conhecimento de aspectos potencialmente úteis e anteriormente inexplorados envolvendo bases de dados ${ }^{19}$.

A etapa subsequente consistiu na avaliação das variáveis relacionadas com as variáveis dependentes através da regressão logística, para mensurar a amplitude das associações. A regressão logística permite estimar diretamente as 
chances de ocorrência de determinada questão a partir de uma variável dependente binária e de um conjunto de variáveis independentes preditoras $^{21}$. As análises procederam-se no software WEKA $^{19}$.

\section{Resultados}

Na Tabela 1 estão expostos os dados quanto à distribuição dos idosos, segundo as variantes formadoras da variável dependente. Considerando as atividades de forma isolada, visualiza-se maior a prevalência de idosos com dificuldade em fazer uso de medicamentos contínuos sem ajuda de terceiros.

A apresentação da distribuição dos idosos, considerando as variáveis utilizadas no estudo que não apresentaram forte relação com as variáveis dependentes, está exposta na Tabela 2.

Nos resultados da análise de seleção de atributos, verificou-se quais as variáveis mais fortemente relacionadas com quadros de DF. A porcentagem expressa por meio do método de validação cruzada de 10 Fold possibilitou a mensuração da ligação entre a variável e o atributo. As variáveis fortemente relacionadas às dificuldade para ABVD foram: idade (100\%); impossibilidade de realizar quaisquer atividades habituais por motivo de saúde (100\%); necessidade de atendimento de emergência no domicílio (100\%); internação hospitalar nos últimos 12 meses (60\%) e condição de saúde geral (50\%). Já as variáveis fortemente relacionadas à dificuldade em AIVD foram: idade (100\%); alfabetização (100\%); vivência com cônjuge (100\%); condição de saúde geral (100\%); presença de alguma doença crônica, física ou mental (100\%); necessidade de atendimento de emergência no domicílio (100\%) e internação hospitalar nos últimos 12 meses (100\%).

Na Tabela 3 é realizada a descrição da amostra de acordo com as variáveis independentes que tiveram relação com a dificuldade funcional.

A análise descritiva mostra que cerca de $3 \%$ e de $4 \%$, respectivamente, dos idosos investigados apresentaram dificuldade para realizar todas as atividades relacionadas às ABVD e AIVD. Os dados ressaltam a elevação na presença de DF envolvendo ABVD na faixa etária acima de 80 anos e AIVD a partir dos 75 anos. Quando o idoso não convive com o cônjuge, a presença de DF eleva-se principalmente na categoria AIVD. Considerando as informações encontradas em relação à escolaridade, destaca-se o montante de idosos não alfabetizados, os quais, quando nesta condição, possuem maior probabilidade de desenvolver DF, com ênfase em AIVD.

Tabela 1. Distribuição dos idosos, segundo as variáveis formadoras das variáveis dependentes ‘DF para ABVD’e 'DF para AIVD', DF independente do grau. Brasil. 2013. $(\mathrm{n}=23.815)$.

\begin{tabular}{lc}
\hline \multicolumn{1}{c}{ Variável dependente e classe } & n (\%) \\
\hline Variáveis formadoras da variável 'Dificuldades ao realizar Atividades Básicas de Vida Diária' & \\
Presença de dificuldade para comer sozinho com um prato colocado à sua frente, incluindo & $1276(5)$ \\
segurar um garfo, cortar alimentos e beber em um copo & $1714(7)$ \\
Presença de dificuldade para tomar banho sozinho incluindo entrar e sair do chuveiro ou banheira & $1555(7)$ \\
Presença de dificuldade para ir ao banheiro sozinho incluindo sentar e levantar do vaso sanitário & $2136(9)$ \\
Presença de dificuldade para se vestir sozinho & $2036(9)$ \\
Presença de dificuldade para andar em casa sozinho de um cômodo a outro da casa & $2040(9)$ \\
Presença de dificuldade para deitar ou levantar da cama sozinho & $1939(8)$ \\
Presença de dificuldade para sentar ou levantar da cadeira sozinho & $4265(18)$ \\
Variáveis formadoras da variável 'Dificuldades ao realizar Atividades Instrumentais de Vida Diária' & $3080(13)$ \\
Presença de dificuldade para fazer compras sozinho & $6852(29)$ \\
Presença de dificuldade para administrar as finanças sozinho & $5659(24)$ \\
Presença de dificuldade para tomar os remédios sozinho & $5453(23)$ \\
Presença de dificuldade para ir ao médico sozinho & $5668(24)$ \\
Presença de dificuldade para sair sozinho utilizando um transporte & \\
Total de idosos livres de DF para todas as atividades &
\end{tabular}

Fonte: Dados da pesquisa.

${ }^{\star}$ Foram considerados para compor a variável dependente apenas indivíduos que apresentaram DF para todas as atividades no rol de ABVD e AIVD, entretanto, tiveram indivíduos que apresentaram mais de uma dependência, porém, não todas. 
Tabela 2. Descrição da amostra, segundo as variáveis dependentes não relacionadas ou menos relacionadas com quadros de DF utilizadas no estudo, segundo idosos independentes, com DF para ABVD, DF para AIVD e total de participantes. Brasil. 2013. $(\mathrm{n}=23.815)$.

\begin{tabular}{|c|c|c|c|c|}
\hline \multirow[t]{2}{*}{ Variáveis Independentes e classes } & $\begin{array}{c}\text { Idosos sem } \\
\text { DF n(\%) }\end{array}$ & $\begin{array}{c}\text { Idosos com } \\
\text { DF em } \\
\text { ABVD n (\%) }\end{array}$ & $\begin{array}{c}\text { Idosos com } \\
\text { DF em } \\
\text { AIVD n }(\%)\end{array}$ & $\begin{array}{c}\text { Tonal n } \\
(\%)\end{array}$ \\
\hline & $\begin{array}{c}\mathrm{N}=22165 \\
(93)\end{array}$ & $\mathrm{N}=649(3)$ & $\mathrm{N}=1001$ & $\begin{array}{c}\mathrm{N}=23815 \\
(100)\end{array}$ \\
\hline \multicolumn{5}{|l|}{ Características Sociodemográficas } \\
\hline \multicolumn{5}{|l|}{ Sexo } \\
\hline Masculino & $12264(55)$ & $265(41)$ & $626(63)$ & $10541(44)$ \\
\hline Feminino & $9901(45)$ & $384(59)$ & $375(37)$ & $13274(56)$ \\
\hline \multicolumn{5}{|l|}{ Cor da pele } \\
\hline Branca & $10266(46)$ & $311(48)$ & $440(44)$ & $11017(46)$ \\
\hline Preta & $1996(9)$ & $62(10)$ & $103(10)$ & $2161(9)$ \\
\hline Amarela & $229(1)$ & $5(1)$ & $6(1)$ & $240(1)$ \\
\hline Parda & $9562(43)$ & $268(41)$ & $449(45)$ & $10279(43)$ \\
\hline Indígena & $108(0)$ & $3(0)$ & $3(0)$ & $114(0)$ \\
\hline Não respondeu & $4(0)$ & --- & --- & $4(0)$ \\
\hline \multicolumn{5}{|l|}{ Estado civil } \\
\hline Casado(a) & $11691(53)$ & $215(33)$ & $310(31)$ & $12216(51)$ \\
\hline Separado(a) ou Divorciado(a) & $1822(8)$ & $38(6)$ & $39(4)$ & $1899(8)$ \\
\hline Viúvo(a) & $5317(24)$ & $285(44)$ & $465(46)$ & $6067(25)$ \\
\hline Solteiro(a) & $3335(15)$ & $111(17)$ & 187 (19) & $3633(15)$ \\
\hline \multicolumn{5}{|l|}{ Formação } \\
\hline Gradu. $\mathrm{Ou}>$ & $8174(37)$ & $239(37)$ & $369(37)$ & $2343(10)$ \\
\hline Médio & $3744(17)$ & $86(13)$ & $104(10)$ & $3253(14)$ \\
\hline Fundamental & $3142(14)$ & $46(7)$ & $65(6)$ & $3934(17)$ \\
\hline Alfabetização & $2297(10)$ & $22(3)$ & $24(2)$ & $8782(37)$ \\
\hline Não respondeu & $4808(22)$ & $256(39)$ & $439(44)$ & $5503(23)$ \\
\hline \multicolumn{5}{|l|}{ Renda familiar mensal } \\
\hline 680 Reais & $9768(44)$ & $394(61)$ & $671(67)$ & $10833(45)$ \\
\hline $681>1320$ reais & $2584(12)$ & $71(11)$ & $94(9)$ & $2749(12)$ \\
\hline $1321>2640$ reais & $2575(12)$ & $82(13)$ & $110(11)$ & $2767(12)$ \\
\hline Mais de 2641 reais & $2713(12)$ & $46(7)$ & $61(6)$ & $2820(12)$ \\
\hline Não informou & $4525(20)$ & $56(9)$ & $65(6)$ & $4646(20)$ \\
\hline \multicolumn{5}{|l|}{ Região de Residência } \\
\hline Norte & $3811(17)$ & $94(14)$ & $162(16)$ & 4067 (17) \\
\hline Nordeste & $6725(30)$ & $260(40)$ & $388(39)$ & $7373(31)$ \\
\hline Sudeste & $6152(28)$ & $153(24)$ & $232(23)$ & $6537(27)$ \\
\hline Sul & $2998(14)$ & $75(12)$ & $107(11)$ & $3180(13)$ \\
\hline Centro-Oeste & $2479(11)$ & $67(10)$ & $112(11)$ & $2658(11)$ \\
\hline \multicolumn{5}{|l|}{ Condição de Saúde } \\
\hline \multicolumn{5}{|c|}{${ }^{\star}$ Esta doença limita de alguma forma suas atividades habituais } \\
\hline $\operatorname{Sim}$ & $3539(44)$ & $396(94)$ & $456(80)$ & $4597(51)$ \\
\hline Não & $4455(56)$ & $27(6)$ & $115(20)$ & $4391(49)$ \\
\hline \multicolumn{5}{|c|}{ *Número de dias deixou de realizar suas atividades habituais, por motivo de saúde } \\
\hline $1-3$ dias & $663(31)$ & $11(4)$ & $34(14)$ & $708(26)$ \\
\hline $4-7$ dias & $594(28)$ & $46(15)$ & $41(17)$ & $681(25)$ \\
\hline $8-11$ dias & $98(5)$ & $4(1)$ & $5(2)$ & $107(4)$ \\
\hline $12-14$ dias & 785 (37) & $237(80)$ & $156(66)$ & $1178(44)$ \\
\hline
\end{tabular}


Tabela 2. Descrição da amostra, segundo as variáveis dependentes não relacionadas ou menos relacionadas com quadros de DF utilizadas no estudo, segundo idosos independentes, com DF para ABVD, DF para AIVD e total de participantes. Brasil. 2013. $(\mathrm{n}=23.815)$.

\begin{tabular}{|c|c|c|c|c|}
\hline \multirow{2}{*}{ Variáveis Independentes e classes } & $\begin{array}{l}\text { Idosos sem } \\
\text { DF n(\%) }\end{array}$ & $\begin{array}{l}\text { Idosos com } \\
\text { DF em } \\
\text { ABVD n }(\%)\end{array}$ & $\begin{array}{l}\text { Idosos com } \\
\text { DF em } \\
\text { AIVD n }(\%)\end{array}$ & $\begin{array}{l}\text { Tonal n } \\
(\%)\end{array}$ \\
\hline & $\begin{array}{c}N=22165 \\
(93)\end{array}$ & $\mathrm{N}=649(3)$ & $\mathrm{N}=1001$ & $\begin{array}{c}\mathrm{N}=23815 \\
(100)\end{array}$ \\
\hline \multicolumn{5}{|l|}{ Precisou ficar acamado nas duas últimas semanas } \\
\hline $\operatorname{Sim}$ & $808(4)$ & $219(34)$ & $126(13)$ & $1153(5)$ \\
\hline Não & $1332(6)$ & $79(12)$ & $110(11)$ & $1521(6)$ \\
\hline Não respondeu & $20025(90)$ & $351(54)$ & $765(76)$ & $21141(89)$ \\
\hline \multicolumn{5}{|c|}{ *Número de dias que esteve acamado nas últimas duas semanas } \\
\hline $1-3$ dias & $335(41)$ & $10(5)$ & $23(18)$ & $368(32)$ \\
\hline $4-7$ dias & $233(29)$ & $26(12)$ & $25(20)$ & $284(25)$ \\
\hline $8-11$ dias & $96(12)$ & $15(7)$ & $13(10)$ & $124(11)$ \\
\hline $12-14$ dias & $144(18)$ & $168(77)$ & $65(52)$ & $377(33)$ \\
\hline \multicolumn{5}{|l|}{ Utilização de Serviços de Saúde } \\
\hline \multicolumn{5}{|c|}{ Local que costuma procurar o primeiro atendimento quando está doente } \\
\hline Farmácia & $432(2)$ & $5(1)$ & $14(1)$ & $451(2)$ \\
\hline UBS & $8159(37)$ & $215(33)$ & $356(36)$ & $8730(37)$ \\
\hline $\begin{array}{l}\text { Centro de especialidades, policlínica pública ou } \\
\text { PAM }\end{array}$ & $326(1)$ & $9(1)$ & $15(1)$ & $350(1)$ \\
\hline $\begin{array}{l}\text { UPA; Pronto Atendimento Público; Pronto- } \\
\text { socorro ou emergência públicos; Hospital público/ } \\
\text { ambulatório/ Pronto-atendimento ou emergência } \\
\text { de hospital privado. }\end{array}$ & $3886(18)$ & $181(28)$ & $215(21)$ & $4282(18)$ \\
\hline $\begin{array}{l}\text { Consultório ou clínica privada; Ambulatório ou } \\
\text { consultório de empresa ou sindicato. }\end{array}$ & $4332(20)$ & $90(14)$ & $169(17)$ & $4591(19)$ \\
\hline No domicilio de forma pública ou privada & $50(0)$ & $12(2)$ & $9(1)$ & $71(0)$ \\
\hline Outro serviço & $86(0)$ & $3(0)$ & $3(0)$ & $92(0)$ \\
\hline Não respondeu & $4894(22)$ & $134(21)$ & $220(22)$ & $5248(22)$ \\
\hline \multicolumn{5}{|l|}{ Costuma procurar o mesmo serviço de saúde sempre } \\
\hline Sim & $17271(78)$ & $515(79)$ & $781(78)$ & $18567(78)$ \\
\hline Não & $4894(22)$ & $134(21)$ & $220(22)$ & $5248(22)$ \\
\hline \multicolumn{5}{|l|}{ Já consultou um profissional médico na vida } \\
\hline $\operatorname{Sim}$ & $22032(99)$ & $649(100)$ & $994(99)$ & $23675(99)$ \\
\hline Não & $133(1)$ & ---- & $7(1)$ & $140(1)$ \\
\hline \multicolumn{5}{|l|}{ Número de consultas no último ano } \\
\hline Até 3 vezes & $9535(43)$ & $195(30)$ & $349(35)$ & $10079(42)$ \\
\hline De 4 a 6 vezes & $5033(23)$ & $184(28)$ & $273(27)$ & $5490(23)$ \\
\hline De 7 a 9 vezes & $898(4)$ & $41(6)$ & $68(7)$ & $1007(4)$ \\
\hline De 10 a 14 vezes & $1886(9)$ & $122(19)$ & $117(12)$ & $2125(9)$ \\
\hline De 15 a 19 vezes & $226(1)$ & $16(2)$ & $28(3)$ & $270(1)$ \\
\hline De 20 a 29 vezes & $296(1)$ & $25(4)$ & $27(3)$ & $348(1)$ \\
\hline 30 ou mais vezes & $145(1)$ & $18(3)$ & $21(2)$ & $184(1)$ \\
\hline Não respondeu & $4146(19)$ & $48(7)$ & $118(12)$ & $4312(18)$ \\
\hline
\end{tabular}

Procurou por algum lugar, serviço ou profissional de saúde para atendimento relacionado à própria saúde nas últimas duas semanas

\begin{tabular}{lrrrr} 
Sim & $5088(23)$ & $220(34)$ & $297(30)$ & $5605(24)$ \\
Não & $17077(77)$ & $429(66)$ & $704(70)$ & $18210(76)$ \\
\hline
\end{tabular}


Tabela 2. Descrição da amostra, segundo as variáveis dependentes não relacionadas ou menos relacionadas com quadros de DF utilizadas no estudo, segundo idosos independentes, com DF para ABVD, DF para AIVD e total de participantes. Brasil. 2013. $(\mathrm{n}=23.815)$.

\begin{tabular}{|c|c|c|c|c|}
\hline \multirow[t]{2}{*}{ Variáveis Independentes e classes } & $\begin{array}{l}\text { Idosos sem } \\
\text { DF n(\%) }\end{array}$ & $\begin{array}{c}\text { Idosos com } \\
\text { DF em } \\
\text { ABVD n (\%) }\end{array}$ & $\begin{array}{c}\text { Idosos com } \\
\text { DF em } \\
\text { AIVD n }(\%)\end{array}$ & $\begin{array}{l}\text { Tonal n } \\
(\%)\end{array}$ \\
\hline & $\begin{array}{c}N=22165 \\
(93)\end{array}$ & $\mathrm{N}=649$ & $\mathrm{~N}=1001$ & $\begin{array}{c}\mathrm{N}=23815 \\
(100)\end{array}$ \\
\hline \multicolumn{5}{|c|}{${ }^{\star}$ Motivo pela procura do atendimento nas duas últimas semanas } \\
\hline Acidente ou lesão & $187(4)$ & $13(6)$ & $11(4)$ & $211(4)$ \\
\hline Doença & $1491(29)$ & $91(41)$ & $99(33)$ & $1681(30)$ \\
\hline Problema odontológico & $96(2)$ & $1(0)$ & $1(0)$ & $98(2)$ \\
\hline Reabilitação ou terapia & $80(2)$ & $9(4)$ & $2(1)$ & $91(2)$ \\
\hline Continuação do tratamento & $1451(29)($ & $70(32)$ & $114(38)$ & $1634(29)$ \\
\hline Exame complementar de diagnóstico & $765(15)$ & $12(5)$ & $34(11)$ & $811(14)$ \\
\hline Vacinação & $18(0)$ & --- & $1(0)$ & $19(0)$ \\
\hline Outro atendimento preventivo & $454(9)$ & $4(2)$ & $15(5)$ & $473(8)$ \\
\hline Solicitação de atestado médico & $10(0)$ & --- & $2(1)$ & $12(0)$ \\
\hline Outro & $536(11)$ & $20(9)$ & $18(6)$ & $575(10)$ \\
\hline \multicolumn{5}{|c|}{${ }^{\star}$ Local onde procurou o último atendimento de saúde nas duas últimas semanas } \\
\hline Farmácia & $24(0)$ & --- & --- & $24(0)$ \\
\hline UBS ou centro de saúde da família & $2076(41)$ & $67(30)$ & $97(33)$ & $2240(40)$ \\
\hline $\begin{array}{l}\text { Centro de especialidades, policlínica pública ou } \\
\text { PAM - Posto de assistência médica. }\end{array}$ & $180(4)$ & $6(3)$ & $9(3)$ & $195(3)$ \\
\hline $\begin{array}{l}\text { UPA, Outro tipo de Pronto Atendimento Público; } \\
\text { Pronto-socorro, ambulatório ou emergência } \\
\text { de hospital público/ Pronto-atendimento ou } \\
\text { emergência de hospital privado }\end{array}$ & $1163(23)$ & $93(42)$ & $109(37)$ & $1365(24)$ \\
\hline $\begin{array}{l}\text { Consultório particular ou clínica privada; } \\
\text { Ambulatório ou consultório de empresa ou sindicato. }\end{array}$ & $1565(31)$ & $33(15)$ & $72(24)$ & $1670(30)$ \\
\hline $\begin{array}{l}\text { No domicílio, com profissional da equipe de saúde } \\
\text { da família; No domicílio, com médico particular }\end{array}$ & $27(1)$ & $18(8)$ & $9(3)$ & $54(1)$ \\
\hline Outro & $53(1)$ & $3(1)$ & $1(0)$ & $57(1)$ \\
\hline \multicolumn{5}{|c|}{ *Teve medicamentos receitados na última consulta (duas últimas semanas) } \\
\hline Não & $3352(66)$ & $157(71)$ & $216(73)$ & $3725(66)$ \\
\hline Sim & $1589(31)$ & $56(25)$ & $72(24)$ & $1717(31)$ \\
\hline Não respondeu & $147(3)$ & $7(3)$ & $9(3)$ & $163(3)$ \\
\hline
\end{tabular}

continua

O modelo de regressão logística teve capacidade explicativa de $79.90 \%$ para ABVD e $76.80 \%$ para AIVD. Na Tabela 4 é possível visualizar as razões de chances para o indivíduo relatar presença de dificuldade ao realizar atividades básicas e instrumentais de vida diária, segundo variáveis independentes.

A Tabela 4 evidencia a elevação das chances dos idosos apresentarem DF na faixa etária dos 80 anos de idade, com aumento gradativo nos anos subsequentes. Indivíduos não alfabetizados apresentaram $\mathrm{OR}=2,23$ mais chances de desenvolve- rem DF para as AIVD. As razões de chances elevaram-se similarmente para ambos os agrupamentos quando o quadro de saúde geral foi considerado ruim. A impossibilidade de realizar qualquer atividade habitual em decorrência do quadro de saúde amplificou em cerca de $\mathrm{OR}=5,56$ as chances de imposição de DF para as ABVD. A necessidade de atendimento emergencial no domicílio ou internação hospitalar mostrou-se relacionada à maior probabilidade de $\mathrm{DF}$ a nível básico $(\mathrm{OR}=$ $3,39$ e $\mathrm{OR}=2,25)$ e instrumental $(\mathrm{OR}=3,21 \mathrm{e} \mathrm{OR}$ $=2,42$ ), respectivamente. 
Tabela 2. Descrição da amostra, segundo as variáveis dependentes não relacionadas ou menos relacionadas com quadros de DF utilizadas no estudo, segundo idosos independentes, com DF para ABVD, DF para AIVD e total de participantes. Brasil. 2013. $(\mathrm{n}=23.815)$.

\begin{tabular}{|c|c|c|c|c|}
\hline \multirow[t]{2}{*}{ Variáveis Independentes e classes } & $\begin{array}{l}\text { Idosos sem } \\
\text { DF n(\%) }\end{array}$ & $\begin{array}{c}\text { Idosos com } \\
\text { DF em } \\
\text { ABVD n (\%) }\end{array}$ & $\begin{array}{c}\text { Idosos com } \\
\text { DF em } \\
\text { AIVD n }(\%)\end{array}$ & $\begin{array}{l}\text { Tonal n } \\
(\%)\end{array}$ \\
\hline & $\begin{array}{c}\mathrm{N}=22165 \\
(93)\end{array}$ & $\mathrm{N}=649(3)$ & $\mathrm{N}=1001$ & $\begin{array}{c}N=23815 \\
(100)\end{array}$ \\
\hline \multicolumn{5}{|c|}{${ }^{\star}$ Qual foi o principal atendimento de saúde que recebeu no último atendimento (duas últimas semanas) } \\
\hline Consulta médica & $3815(75)$ & $138(63)$ & $217(73)$ & $4162(74)$ \\
\hline Consulta odontológica & $75(1)$ & $1(0)$ & $1(0)$ & $77(1)$ \\
\hline $\begin{array}{l}\text { Consulta com outro profissional de saúde (fisio, } \\
\text { fono, pscó, nutri, enf, etc.) }\end{array}$ & $329(6)$ & $19(9)$ & $19(6)$ & $367(7)$ \\
\hline Atendimento com agente comunitário de saúde & $20(0)$ & $5(2)$ & $1(0)$ & $26(0)$ \\
\hline Atendimento na farmácia & $21(0)$ & --- & --- & $21(0)$ \\
\hline Vacinação & $22(0)$ & $2(1)$ & $1(0)$ & $25(0)$ \\
\hline Injeção, curativo ou medição de pressão arterial & $87(2)$ & $2(1)$ & $10(3)$ & $99(2)$ \\
\hline $\begin{array}{l}\text { Quimioterapia, radioterapia, hemodiálise ou } \\
\text { hemoterapia }\end{array}$ & $34(1)$ & $3(1)$ & $8(3)$ & $45(1)$ \\
\hline $\begin{array}{l}\text { Exames laboratoriais ou de imagem ou exames } \\
\text { complementares de diagnóstico }\end{array}$ & $274(5)$ & $8(4)$ & $10(3)$ & $292(5)$ \\
\hline Gesso ou imobilização & $12(0)$ & $4(2)$ & $1(0)$ & $17(0)$ \\
\hline Pequena cirurgia ambulatorial & $46(1)$ & $2(1)$ & $3(1)$ & $51(1)$ \\
\hline Internação hospitalar & $52(1)$ & $16(7)$ & $13(4)$ & $81(1)$ \\
\hline Marcação de consulta & $13(0)$ & $13(6)$ & $1(0)$ & $19(0)$ \\
\hline $\begin{array}{l}\text { Práticas complementares como acupuntura, } \\
\text { homeopatia e fitoterapia }\end{array}$ & $11(0)$ & ---- & $12(4)$ & $11(0)$ \\
\hline Outro atendimento (especifique: & $143(3)$ & --- & --- & $168(3)$ \\
\hline Não respondeu & $134(3)$ & $7(3)$ & --- & $144(3)$ \\
\hline \multicolumn{5}{|c|}{${ }^{\star} \mathrm{O}$ serviço de saúde em que procurou atendimento nas duas últimas semanas era } \\
\hline Público & $3036(60)$ & $156(71)$ & $194(65)$ & $3386(60)$ \\
\hline Privado & $1913(38)$ & $57(26)$ & $93(31)$ & $2063(37)$ \\
\hline Não sabe & $9(0)$ & --- & $2(1)$ & $12(0)$ \\
\hline Não respondeu & $130(3)$ & $7(3)$ & $8(3)$ & $144(3)$ \\
\hline \multicolumn{5}{|c|}{ Utilizou alguma prática integrativa e complementar nos 12 últimos meses } \\
\hline Sim & $1327(6)$ & $37(6)$ & $56(6)$ & $1420(6)$ \\
\hline Não & $20838(94)$ & $612(94)$ & $945(94)$ & $22395(94)$ \\
\hline \multicolumn{5}{|l|}{ Internações e Urgências Médicas } \\
\hline \multicolumn{5}{|c|}{${ }^{\star}$ Nos últimos 12 meses, quantas vezes esteve internado (a) } \\
\hline $1-3$ vezes & $1847(95)$ & $170(80)$ & $193(86)$ & $2210(93)$ \\
\hline $4-6$ vezes & $75(4)$ & $29(14)$ & $26(12)$ & $130(5)$ \\
\hline $7-9$ vezes & $9(0)$ & $3(1)$ & $1(0)$ & $13(1)$ \\
\hline $10-14$ vezes & $9(0)$ & $10(5)$ & $5(2)$ & $24(1)$ \\
\hline
\end{tabular}

${ }^{\star}$ Qual foi o principal atendimento de saúde que recebeu quando esteve internado (a) (pela última vez) nos doze últimos meses

Tratamento clínico

Tratamento psiquiátrico

$826(43)$

$126(59)$

$135(60) \quad 1087(46)$

Cirurgia

$15(1)$

$1(0)$

$3(1)$

$19(1)$

Exames complementares

685 (35)

35 (17)

35 (16)

754 (32)

Outro

117 (6)

$14(7)$

$19(8)$

150 (6)

297 (15)

36 (17)

$33(15)$

366 (15) 
Tabela 2. Descrição da amostra, segundo as variáveis dependentes não relacionadas ou menos relacionadas com quadros de DF utilizadas no estudo, segundo idosos independentes, com DF para ABVD, DF para AIVD e total de participantes. Brasil. 2013. $(\mathrm{n}=23.815)$.

\begin{tabular}{|c|c|c|c|c|}
\hline \multirow[t]{2}{*}{ Variáveis Independentes e classes } & $\begin{array}{c}\text { Idosos sem } \\
\text { DF n(\%) }\end{array}$ & $\begin{array}{c}\text { Idosos com } \\
\text { DF em } \\
\text { ABVD n (\%) }\end{array}$ & $\begin{array}{c}\text { Idosos com } \\
\text { DF em } \\
\text { AIVD n }(\%)\end{array}$ & $\begin{array}{c}\text { Tonal n } \\
(\%)\end{array}$ \\
\hline & $\begin{array}{c}\mathrm{N}=22165 \\
(93)\end{array}$ & $\mathrm{N}=649$ & $\mathrm{~N}=1001$ & $\begin{array}{c}\mathrm{N}=23815 \\
(100)\end{array}$ \\
\hline \multicolumn{5}{|l|}{${ }^{*}$ Quanto tempo ficou internada(a) na última vez } \\
\hline $1-3$ dias & $1884(97)$ & $165(78)$ & $198(88)$ & $2247(95)$ \\
\hline $4-6$ dias & $11(1)$ & $22(10)$ & $15(7)$ & $48(2)$ \\
\hline $7-9$ dias & $5(0)$ & $4(2)$ & $2(1)$ & $11(0)$ \\
\hline $10-14$ dias & $6(0)$ & $1(0)$ & --- & $7(0)$ \\
\hline $15-19$ dias & --- & $1(0)$ & --- & $1(0)$ \\
\hline 30 dias ou mais & $34(2)$ & $19(9)$ & $10(4)$ & $63(3)$ \\
\hline \multicolumn{5}{|c|}{${ }^{\star} \mathrm{Na}$ última vez que foi internado como foi o atendimento recebido } \\
\hline Bom & $1687(87)$ & $164(77)$ & $183(81)$ & $2034(86)$ \\
\hline Ruim & $253(13)$ & $48(23)$ & $42(19)$ & $343(14)$ \\
\hline \multicolumn{5}{|c|}{${ }^{\star}$ Na última vez que teve atendimento de urgência no domicílio, como foi o atendimento recebido } \\
\hline Bom & $374(87)$ & $106(87)$ & $109(87)$ & $589(87)$ \\
\hline Ruim & $54(13)$ & $16(13)$ & $16(13)$ & $86(13)$ \\
\hline \multicolumn{5}{|c|}{${ }^{\star}$ Neste atendimento foi transportado por ambulância para um serviço de saúde } \\
\hline Sim & $238(56)$ & $76(62)$ & $85(68)$ & $399(59)$ \\
\hline Não & $190(44)$ & $46(38)$ & $40(32)$ & $276(41)$ \\
\hline \multicolumn{5}{|l|}{ Condição de Saúde Bucal } \\
\hline \multicolumn{5}{|l|}{ Autopercepção de saúde bucal } \\
\hline Boa & $6486(29)$ & $74(11)$ & $174(17)$ & $6734(28)$ \\
\hline Ruim & $4073(18)$ & $149(23)$ & $221(22)$ & $4443(19)$ \\
\hline Não respondeu & $11606(52)$ & $426(66)$ & $606(61)$ & $12638(53)$ \\
\hline \multicolumn{5}{|c|}{ Dificuldade para comer em decorrência de problemas dentários } \\
\hline Não tem nenhuma dificuldade & $8352(38)$ & $223(34)$ & $229(23)$ & $8686(36)$ \\
\hline Tem dificuldade & $2207(10)$ & $118(18)$ & $165(16)$ & $2491(10)$ \\
\hline Não respondeu & $11606(52)$ & $308(47)$ & $607(61)$ & $12638(53)$ \\
\hline \multicolumn{5}{|l|}{ Perda dental inferior } \\
\hline Não & $901(4)$ & $8(1)$ & $25(2)$ & $934(4)$ \\
\hline Sim, perdi & $5274(24)$ & $67(10)$ & $135(13)$ & $5474(23)$ \\
\hline Sim, perdi todos os dentes de baixo & $4384(20)$ & $148(23)$ & $235(23)$ & $4767(20)$ \\
\hline Não responderam & $11606(52)$ & $426(66)$ & $606(61)$ & $12640(53)$ \\
\hline \multicolumn{5}{|l|}{ Perda dental superior } \\
\hline Não & $747(3)$ & $9(1)$ & $16(2)$ & $772(3)$ \\
\hline Sim, perdi & $3957(18)$ & $45(7)$ & $101(10)$ & $4104(17)$ \\
\hline Sim, perdi todos os dentes de cima & $5855(26)$ & $169(26)$ & $278(28)$ & $6302(26)$ \\
\hline Não respondeu & $11606(52)$ & $426(66)$ & $606(61)$ & $12637(53)$ \\
\hline \multicolumn{5}{|l|}{ Número de dentes naturais presentes } \\
\hline Menos de 10 dentes & $16370(74)$ & $577(89)$ & $850(85)$ & $17797(75)$ \\
\hline Mais de 10 dentes & $5795(26)$ & $72(11)$ & $151(15)$ & $6018(25)$ \\
\hline \multicolumn{5}{|l|}{ Uso de prótese dentária } \\
\hline Não & $2740(12)$ & $98(15)$ & $173(17)$ & $3011(13)$ \\
\hline $\begin{array}{l}\text { Próteses dentárias totais em uma das arcadas ou } \\
\text { para substituir determinado número de dentes }\end{array}$ & $3983(18)$ & $52(8)$ & $79(8)$ & $4115(17)$ \\
\hline Próteses dentárias totais para ambas as arcadas & $3404(15)$ & $68(10)$ & $132(13)$ & $3603(15)$ \\
\hline Não respondeu & $12038(54)$ & $431(66)$ & $617(62)$ & $13086(55)$ \\
\hline \multicolumn{5}{|l|}{ Consultou um cirurgião dentista alguma vez na vida } \\
\hline Sim & $21110(95)$ & $579(89)$ & $900(90)$ & $22589(95)$ \\
\hline Não & $1055(5)$ & $70(11)$ & $101(10)$ & $1226(5)$ \\
\hline
\end{tabular}

Fonte: Dados da pesquisa.

${ }^{\star}$ Calculado percentual baseado no número de indivíduos que necessitaram de determinado atendimento e ou passaram pela situação em questão. 
Tabela 3. Descrição das variáveis fortemente com a DF, para idosos independentes, com DF para ABVD, DF para AIVD e totalidade de respondentes. Brasil. 2013. $(n=23.815)$.

\begin{tabular}{|c|c|c|c|c|c|c|c|c|}
\hline \multirow{3}{*}{ Variáveis Independentes e classes } & \multicolumn{2}{|c|}{ Idosos sem DF } & \multicolumn{2}{|c|}{$\begin{array}{c}\text { Idosos com DF } \\
\text { em ABVD }\end{array}$} & \multicolumn{2}{|c|}{$\begin{array}{c}\text { Idosos com DF } \\
\text { em AIVD }\end{array}$} & \multicolumn{2}{|c|}{ Total de idosos } \\
\hline & $\mathbf{n}$ & $\%$ & $\mathbf{n}$ & $\%$ & $\mathbf{n}$ & $\%$ & $\mathbf{n}$ & $\%$ \\
\hline & 22165 & 93 & 649 & 3 & 1001 & 4 & 23815 & 100 \\
\hline \multicolumn{9}{|l|}{ Idade } \\
\hline 60 a 64 anos & \multicolumn{2}{|c|}{$7590(34)$} & \multicolumn{2}{|c|}{$63(10)$} & \multicolumn{2}{|c|}{$98(10)$} & \multicolumn{2}{|c|}{$7751(33)$} \\
\hline 65 a 69 anos & \multicolumn{2}{|c|}{$5595(25)$} & \multicolumn{2}{|c|}{$61(9)$} & \multicolumn{2}{|c|}{$110(11)$} & \multicolumn{2}{|c|}{$5766(24)$} \\
\hline 70 a 74 anos & \multicolumn{2}{|c|}{$4022(18)$} & \multicolumn{2}{|c|}{$86(13)$} & \multicolumn{2}{|c|}{$119(12)$} & \multicolumn{2}{|c|}{$4227(18)$} \\
\hline 75 a79 anos & \multicolumn{2}{|c|}{$2579(12)$} & \multicolumn{2}{|c|}{$98(15)$} & \multicolumn{2}{|c|}{$165(16)$} & \multicolumn{2}{|c|}{$2842(12)$} \\
\hline 80 a 84 anos & \multicolumn{2}{|c|}{$1433(6)$} & \multicolumn{2}{|c|}{$120(18)$} & \multicolumn{2}{|c|}{$222(22)$} & \multicolumn{2}{|c|}{$1775(7)$} \\
\hline 86 a 89 anos & \multicolumn{2}{|c|}{$669(3)$} & \multicolumn{2}{|c|}{$111(17)$} & 160 & & & (4) \\
\hline 90 a 94 anos & & (1) & & & & & & (2) \\
\hline 95 a 100 anos & & (0) & & & & & & (0) \\
\hline 100 ou mais anos & & $(0)$ & & & & & & $(0)$ \\
\hline Convive com cônjuge ou companhei & & & & & & & & \\
\hline Sim & 12875 & 85) & 232 & & 336 & & 13443 & 56) \\
\hline Não & 9290 & 42) & 417 & & 665 & & 10372 & 44) \\
\hline Alfabetizado & & & & & & & & \\
\hline Sim & 17058 & 77) & 389 & & 538 & & 17985 & 76) \\
\hline Não & 5107 & 23) & 260 & & 463 & & 5830 & 24) \\
\hline Avaliação do estado de saúde geral & & & & & & & & \\
\hline Bom & 10182 & 46) & & & 207 & & 10463 & 44) \\
\hline Ruim & 11983 & 54) & 575 & & 794 & & 13352 & 56) \\
\hline Deixou de realizar quaisquer de suas & tividades & labitua & $\mathrm{s}$ por $\mathrm{m}$ & vo de $s$ & úde nas & as últir & as seman & \\
\hline Não & 20025 & 90) & 351 & & & & 21141 & 89) \\
\hline Sim & 2140 & 10) & 298 & & & & 2674 & 11) \\
\hline $\begin{array}{l}\text { Algum médico já lhe diagnosticou cc } \\
\text { (superior a seis meses) }\end{array}$ & $n$ algume & pença & crônica, & ica ou & lental, o & oença & longa & ração \\
\hline Não & 7994 & 36) & 423 & & 571 & & 8988 & 38) \\
\hline Sim & 14171 & 64) & 226 & & 430 & & 14827 & 62) \\
\hline Nos últimos 12 meses, precisou de at & ndiment & de eme & gência & domicí & & & & \\
\hline Não & & & & & & & 23140 & 97) \\
\hline Sim & & & 123 & & 125 & & & (3) \\
\hline Nos últimos 12 meses, ficou internac & (a) em l & spital & or $24 \mathrm{~h}$ & is ou $\mathrm{m}$ & & & & \\
\hline Não & 20225 & 91) & 437 & & 776 & & 21438 & 90) \\
\hline Sim & 194 & (9) & 212 & & 225 & & 2377 & 10) \\
\hline
\end{tabular}

Fonte: Dados da pesquisa.

\section{Discussão}

As demandas em saúde oriundas da população idosa percorrem um caminho inconstante composto por inúmeras barreiras e dificuldades. No campo da saúde, a carência de acesso e apreensão de informações, a falta de estrutura física, de investimento e de capacitação profissional, entre outros, caracterizam-se como responsáveis pela impossibilidade de planejamento e desenvolvimento de estratégias contínuas e efetivas e, consequentemente, pela ampliação das marcas físicas e psicológicas geradas pelo tempo.
Prosseguindo-se nos conhecimentos de saúde da população idosa brasileira, dá-se valor à importação de achados relacionados à presença de DF publicados em estudos prévios. Uma pesquisa, ao analisar 388 idosos com idade média de 71 anos, deparou-se com a presença de DF para realização de ABVD em 12,6\% dos participantes, e na execução de AIVD, em 45,6\% da população ${ }^{7}$. Outra análise, realizada com 1.624 idosos residentes na região metropolitana de Belo Horizonte-MG, apontou que aproximadamente um terço dos participantes possuía algum tipo de DF, sendo mais prevalentes as dificuldades para AIVD $^{3}$. 
Tabela 4. Razões de chances da presença de dificuldade ao realizar Atividades Básicas e Instrumentais de Vida Diária, segundo variáveis independentes.

\begin{tabular}{|c|c|c|}
\hline Variável & $\begin{array}{c}\text { Oddis Ratio (OR) } \\
\text { ABVD }\end{array}$ & $\begin{array}{c}\text { Oddis Ratio (OR) } \\
\text { AIVD }\end{array}$ \\
\hline \multicolumn{3}{|l|}{ Idade } \\
\hline 60 a 64 anos & 0,23 & 0,30 \\
\hline 65 a 69 anos & 0,33 & 0,41 \\
\hline 70 a 74 anos & 0,59 & 1,00 \\
\hline 75 a79 anos & 1,00 & 1,10 \\
\hline 80 a 84 anos & 2,25 & 2,81 \\
\hline 86 a 89 anos & 5,28 & 4,39 \\
\hline 90 a 94 anos & 10,07 & 7,97 \\
\hline 95 a 100 anos & 11,38 & 9,55 \\
\hline Mais de 100 anos & 66,31 & 21,76 \\
\hline \multicolumn{3}{|l|}{ Alfabetizado } \\
\hline $\operatorname{Sim}$ & -- & 1,00 \\
\hline Não & -- & 2,23 \\
\hline \multicolumn{3}{|l|}{ Convive com o cônjuge ou companheiro } \\
\hline Sim & -- & 1,00 \\
\hline Não & -- & 1,85 \\
\hline \multicolumn{3}{|l|}{ Percepção da condição de saúde geral } \\
\hline Boa & 1,00 & 1,00 \\
\hline Ruim & 4,77 & 2,86 \\
\hline \multicolumn{3}{|c|}{$\begin{array}{l}\text { Impossibilidade de realizar qualquer uma das atividades habituais por motivo de saúde (duas últimas } \\
\text { semanas) }\end{array}$} \\
\hline Não & 1,00 & -- \\
\hline $\operatorname{Sim}$ & 5,56 & -- \\
\hline \multicolumn{3}{|c|}{ Diagnóstico de alguma doença crônica, física ou mental } \\
\hline Não & -- & 1,00 \\
\hline $\operatorname{Sim}$ & -- & 1,98 \\
\hline \multicolumn{3}{|c|}{ Necessidade de atendimento de emergência no domicílio nos últimos 12 meses } \\
\hline Não & 1,00 & 1,00 \\
\hline $\operatorname{Sim}$ & 3,39 & 3,21 \\
\hline \multicolumn{3}{|c|}{ Necessidade de internação hospitalar nos últimos 12 meses } \\
\hline Não & 1,00 & 1,00 \\
\hline Sim & 2,25 & 2,42 \\
\hline
\end{tabular}

Fonte: Dados da pesquisa.

No presente estudo, os achados demostraram a presença de DF para realização de todas as ABVD e AIVD, com frequências de $3 \%$ e $4 \%$, respectivamente. Esta atenuação dos valores da prevalência de DF, considerando-se os dados da PNS vigente e os de literatura brasileira atual, pode estar relacionada à delimitação metodológica diferenciada considerada para o presente estudo $^{2}$, a qual classifica com DF os indivíduos com dificuldade total para as atividades básicas e instrumentais.

O declínio funcional tem início ainda na terceira década de vida, ganhando velocidade com o passar dos anos, sendo influenciado por aspec- tos biopsicossociais e pela presença de doenças, principalmente as crônicas não transmissíveis (DCNT) $)^{1,7,8,22}$. Esta aceleração temporal do processo de declínio funcional torna-se evidente a partir dos dados expostos na Tabela 3, com destaque para a ascensão da probabilidade de desenvolver DF a partir dos 80 anos.

Destaca-se que na Classificação Internacional de Funcionalidade, Incapacidade e Saúde (CIF), medida instituída pela Organização Mundial de Saúde ${ }^{23}$, essa abordagem biopsicossocial para a funcionalidade de indivíduos idosos é igualmente considerada. Na referida classificação, dois componentes são destacados: os fatores de 
funcionalidade e incapacidade, e os contextuais. O primeiro agrupamento refere-se às funções físicas, estruturas corporais, atividades e atuação (no contexto individual e social), e o segundo envolve aspectos ambientais e pessoais ${ }^{6,23}$.

Estudo demonstra que o surgimento da DF habitualmente percorre o caminho das AIVD em direção às $\mathrm{ABVD}^{12}$, em consonância com os achados da Tabela 2. Ainda, que as primeiras dificuldades que se instauram na rotina do indivíduo são as relacionadas à depredação de sua condição cognitiva, sendo esta pertencente ao rol de $\mathrm{AIVD}^{12}$. Os achados expostos remetem à necessidade de alerta no campo da saúde, valendo-se do indicador relacionado à DF como base para planejamentos específicos, visando à redução dos casos de dependência para desempenhar as atividades instrumentais e básicas. Nesse sentido, ressalta-se a importância de desempenhar ações acessíveis envolvendo a promoção e a prevenção da saúde de indivíduos com 60 ou mais anos, em consonância com o exposto na Política Nacional de Saúde da Pessoa Idosa ${ }^{24}$.

O contexto abordado no presente estudo mostrou-se influenciado por múltiplos aspectos. O nível de escolaridade apresentou-se como um deles; quando não alfabetizado, o indivíduo idoso possuía mais possibilidades de desenvolver DF para AIVD $(\mathrm{OR}=2,23)$ (Tabela 4). Geralmente indivíduos não alfabetizados são submetidos a condições de trabalho menos favoráveis, além de terem menor oportunização em relação ao acesso a ações preventivas em serviços de saúde ${ }^{7}$. Os níveis de escolaridade além de contribuírem na oportunização laboral, possuem direta ligação com as condições de vida, situação econômica, moradia, alimentação e lazer, modulando o estilo de vida a ser seguido pelo idoso, as oportunidades e as relações sociais, interferindo concomitantemente em aspectos psicológicos ${ }^{1}$.

Outro aspecto importante associado à DF nos idosos estudados foi o suporte decorrente da convivência com companheiro(a), apresentando influência direta para o agrupamento de DF para as AIVD. Independente da qualidade desta relação de convívio, quando o idoso não dividia a residência com um(a) companheiro(a), apresentou cerca de $\mathrm{OR}=1,85$ mais chances de desenvolver DF. A situação relaciona-se com aspectos emocionais, como sentimento de isolamento e dependência ${ }^{7}$, assim como, com aspectos práticos da vida diária. Como exemplo, sabe-se que entre mulheres idosas, dividir moradia constitui-se um fator influenciador na diminuição da procura por serviços de saúde para o tratamento de doença ${ }^{5}$. A relação com o cônjuge constitui-se como preferencial no sentido de auxílio e apoio, e estimula a participação social e a manutenção das capacidades funcionais ${ }^{25,26}$.

A demanda pela busca por serviços de saúde entre idosos cresceu nos últimos anos no Brasil, como mostram os dados de outro inquérito nacional de base populacional, a Pesquisa Nacional por Amostra de Domicílio (PNAD). Na PNAD de 1998, 2003 e 2008 evidencia-se que a procura de serviços de saúde por idosos para o tratamento de doenças ou situações agudas passou de $8 \%$ para $13 \%$, enquanto a busca por tratamentos preventivos ou de rotina caiu de $9 \%$ pra $5 \%$.

$\mathrm{O}$ aspecto em debate remete a inexoráveis limitações de saúde apresentadas para a população idosa brasileira, sobre as quais o sistema público de saúde não vem suprindo a sólida demanda existente, ocasionando grande parcela de indivíduos insatisfeitos com a própria condição de saúde. Conforme os dados, quando o idoso relatou a saúde geral como ruim, a possibilidade de desenvolver DF para $\mathrm{ABVD}(\mathrm{OR}=4,77)$ e para $\mathrm{AIVD}$ $(\mathrm{OR}=2,86)$ elevou-se.

A presença de doenças está associada a piores condições funcionais e consequente percepção negativa da própria situação ${ }^{27,28}$. A autopercepção em relação à saúde é modulada por inúmeras questões, incluindo as dimensões biológica, psicológica e social, sendo muitas vezes ignorada por profissionais e pesquisadores. A falta de conhecimento sobre a integração dinâmica entre essas dimensões, e o entendimento de que o estado geral de saúde admite igualmente a influência de questões físicas e da subjetividade do indivíduo, podem, então, impactar negativamente em tomadas assertivas de decisão para a população idosa.

A capacidade funcional é considerada um processo dinâmico, configurando-se como elemento chave na saúde do idoso ${ }^{1,3,29}$. Por outro lado, a DF é associada a quadros negativos de saúde ou torna-se propulsora destes entre ido$\operatorname{sos}^{1,3,29}$, culminando em maior necessidade de

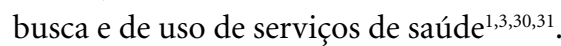

A impossibilidade de realizar quaisquer das atividades habituais devido a algum motivo de saúde elevou as chances do idoso apresentar DF para $\mathrm{ABVD}(\mathrm{OR}=5,56)$, enquanto que a presença de alguma doença crônica (física ou mental) adicionou probabilidades de conviver com DF para as AIVD $(\mathrm{OR}=1,98)$.

Outra associação pertinente com quadros de DF foi a necessidade de atendimento emergencial domiciliar. Ao vivenciar uma situação dentro desse agrupamento temático, o idoso apresentou OR 
$=3,39$ e $\mathrm{OR}=3,21$ mais chances de apresentar DF para ABVD e AIVD, respectivamente. Estudos mencionam a associação de quadros de DF com a necessidade de atendimento no domicílio devido a restrições de locomoção, a qual pode ocasionar em quedas ${ }^{1,3}$ e em situações críticas de saúde, expondo o indivíduo a maiores riscos e, consequentemente, a cuidados de maior complexidade ${ }^{32}$. Na presença de doenças, com destaque para as crônicas, existe maior propensão de uso de serviços de emergência, em decorrência da evolução do caso e das restriçõos originadas, como a impossibilidade de realizar atividades habituais e dificuldades funcionais para $\mathrm{ABVD}$ e $\mathrm{AIVD}^{33}$.

Os dados considerados mostraram ainda que os idosos que estiveram internados expuseram uma conjuntura mais propícia para desenvolver DF, para ambos os grupos: $\mathrm{ABVD}$ OR $=2,25 \mathrm{e}$ AIVD $O R=2,42$. A principal justificativa para tal resultado consiste no agravamento do desempenho funcional do idoso durante o período de internação ${ }^{34-36}$. As limitações decorrentes da DF para ambas as categorias (ABVD e AIVD) oneram maior utilização dos serviços de saúde e indicam quadros mais complexos, os quais podem culminar na internação hospitalar ${ }^{1}$.

Culturalmente, a população brasileira associa idosos a restrições físicas e psicológicas, interpretando-as como consequência natural do envelhecimento. A ascensão da expectativa de vida e do número de idosos deve ser estrategicamente aguardada com medidas que incluam atividades físicas, recreativas e hábitos saudáveis na rotina de vida desses sujeitos, e com medidas propulsoras de relações sociais, da preservação de capacidades físicas e psíquicas, evitando agravamento de condições limitantes instauradas e declínio funcional. Com base nas recomendações dos achados do presente estudo e em consonância com as normativas nacionais para a pessoa idosa, destacam-se ações promotoras de saúde que já vêm sendo desenvolvidas no país, como: academias da saúde, criação de ambientes de ensino para a terceira idade, espaços de convivência intergeracional (incluindo jogos, danças, e outras ações) e estímulo para a busca preventiva por serviços de saúde ${ }^{37}$.

Finaliza-se afirmando que o processo fisiológico do envelhecimento não pode ser evitado, entretanto, ações voltadas à prevenção e promoção da saúde podem retardar o seu desenvolvimento e minimizar suas expressões nos níveis físico e psicológico, oportunizando ao sujeito idoso qualidade em múltiplos aspectos da longevidade. Fomenta-se a importância do conhecimento, das investigações, da constante necessidade de inovação na forma de desenvolver ações na área da saúde e da demanda imediata de efetivação do trabalho em equipe de forma interdisciplinar.

\section{Limitações do estudo}

Considerando inicialmente a fonte deste estudo, ou seja, os dados da Pesquisa Nacional de Saúde vigente, uma primeira restrição refere-se ao fato de que as informações poderiam ter sido fornecidas por moradores distintos do domićlío, e não necessariamente pelo próprio idoso. Entretanto, estudos prévios publicados reforçam a premissa de que tal situação não desvaloriza os dados ${ }^{38}$.

As questões abordadas no questionário da PNS relacionadas às ABVD e AIVD não contemplavam todos os parâmetros frequentemente investigados através dos renomados instrumentos como: Índice de Katz para investigar a capacidade funcional relacionada às ABVD e a Escala de Lawton e Brody para AIVD ${ }^{1}$.

Especificamente, em relação ao estudo proposto, deve-se ter um olhar cauteloso junto aos dados conformadores das variáveis dependentes, uma vez que foram considerados como indivíduos portadores de DF apenas aqueles que a apresentavam em todas as variáveis formadoras das variáveis dependentes, independente do grau de acometimento. Deste modo, não foi possível reconhecer as associações relacionadas aos indivíduos que perderam apenas algumas das funções e em graus definidos. Contudo, esta limitação não minimiza a importância do estudo, uma vez que se buscou demonstrar a avaliação de cenários mais desfavoráveis possíveis, para ambas as $\mathrm{DF}$ em AIVD e ABVD, uma vez que esses cenários exigem o maior número de esforços possíveis perante a família, a sociedade e o governo.

\section{Conclusões}

A interpretação dos dados extraídos reforça associações já consolidadas pela literatura pertinente, as quais permitem o fortalecimento do planejamento e da execução de estratégias às demandas relacionadas à população idosa brasileira, além da orientação de investimentos para ações voltadas à preservação e à manutenção de capacidades físicas e biopsicossociais emocionais de idosos, possibilitando o seu empoderamento e protagonismo em relação à própria saúde.

Desta forma, os resultados do presente estudo devem contribuir de forma significativa para o planejamento e o desenvolvimento de um con- 
junto articulado e contínuo de ações e serviços preventivos e curativos de saúde direcionados, por gestores de políticas públicas, aos sujeitos idosos no Brasil. Ainda, os dados evidenciam a necessidade da priorização de ações destinadas a indivíduos acima de 80 anos, com fomento à participação social, familiar e de aporte profissional multidisciplinar no campo da saúde.

\section{Colaboradores}

C Zanesco contribuiu na concepção, delineamento, análise e interpretação dos dados, redação do artigo. D Bordin contribuiu na concepção, delineamento, análise e interpretação dos dados, redação do artigo, revisão crítica e aprovação da versão submetida do artigo. CB Santos contribuiu no delineamento, análise e interpretação dos dados. CB Fadel contribuiu na concepção, delineamento, análise e interpretação dos dados, redação do artigo, revisão crítica e aprovação da versão submetida do artigo.

\section{Referências}

1. Nunes JD, Saes MDO, Nunes BP, Siqueira FCV, Soares DC, Fassa MEG, Thumé E, Facchini LA, Nunes JD, Saes MDO, Nunes BP, Siqueira FCV, Soares DC, Fassa MEG, Thumé E, Facchini LA. Indicadores de incapacidade funcional e fatores associados em idosos: estudo de base populacional em Bagé, Rio Grande do Sul. Epidemiol Serv Saude 2017; 26(2):295-304.

2. Brito KQD, Menezes TN, Olinda RAD. Incapacidade funcional: condições de saúde e prática de atividade física em idosos. Rev Bras Enferm 2016; 69(5):825-832.

3. Fialho CB, Lima-Costa MF, Giacomin KC, Loyola Filho AID. Capacidade funcional e uso de serviços de saúde por idosos da Região Metropolitana de Belo Horizonte, Minas Gerais, Brasil: um estudo de base populacional. Cad Saude Publica 2014; 30(3):599-610.

4. Oliveira-Figueiredo DSTD, Felisbino-Mendes MS, Malta DC, Velasquez-Melendez G. Prevalence of functional disability in the elderly: analysis of the National Health Survey. Rev RENE 2017; 18(4):468-475.

5. Almeida AND. O acesso aos serviços de saúde pelos idosos no Brasil com base na Pesquisa Nacional por Amostra de Domicílios (PNAD) entre 1998 e 2008. J Bras Econ Saude 2015; 7(1):43-52.

6. Lopes GL, Santos MIPDO. Funcionalidade de idosos cadastrados em uma unidade da Estratégia Saúde da Família segundo categorias da Classificação Internacional de Funcionalidade. Rev Bras Geriatr e Gerontol 2015; 18(1):71-83.

7. Pereira LC, Figueiredo MDLF, Beleza CMF, Andrade EMLR, Silva MJD, Pereira AFM. Fatores preditores para incapacidade funcional de idosos atendidos na atenção básica. Rev Bras Enferm 2017; 70(1):112-118.

8. Virtuoso-Júnior JS, Tribessa S, Menezes AS, Meneguci J, Sasakia JE. Fatores associados à incapacidade funcional em idosos brasileiros. Rev Andal Med Deport 2016; 1(1):1-7.

9. Campos ACV, Almeida MHMD, Campos GV, Bogutchi TF. Prevalência de incapacidade funcional por gênero em idosos brasileiros: uma revisão sistemática com metanálise. Rev Bras Geriatr e Gerontol 2016; 19(3):545-559.

10. Pilger C, Menon MU, Mathias TADF. Utilização de serviços de saúde por idosos vivendo na comunidade. Rev Esc Enferm USP 2013; 47(1):213-220. 
11. Malta D, Andrade, Stopa S, Pereira C, Szwarcwald C, Júnior JBDS, Reis AACD. Estilos de vida da população brasileira : resultados da Pesquisa Nacional de Saúde, 2013. Epidemiol Serv Saude 2015; 24(2):217-226.

12. Barbosa BR, Almeida JMD, Barbosa MR, Rossi-Barbosa LAR. Avaliação da capacidade funcional dos idosos e fatores associados à incapacidade. Cien Saude Colet 2014; 19(8):3317-3325.

13. Szwarcwald CL, Malta DC, Pereira CA, Vieira MLFP, Conde WL, Souza Júnior PRBD, Damacena GN, Azevedo LO, Azevedo e Silva G, Theme Filha MM, Lopes CDS, Romero DE, Almeida WDSD, Monteiro CA. Pesquisa Nacional de Saúde no Brasil: concepção e metodologia de aplicação. Cien Saude Colet 2014; 19(2):333-342.

14. Souza-Júnior PRBD, Freitas MPSD, Antonaci GDA, Szwarcwald CL. Desenho da amostra da Pesquisa Nacional de Saúde 2013. Epidemiol Serv Saude 2015; 24(2):207-216.

15. Damacena GN, Szwarcwald CL, Malta DC, Souza Júnior PRBD, Vieira MLFP, Pereira CA, Morais Neto OLD, Silva Júnior JBD. O processo de desenvolvimento da Pesquisa Nacional de Saúde no Brasil, 2013. Epidemiol Serv Saude 2015; 24(2):197-206.

16. Instituto Brasileiro de Geografia e Estatística (IBGE). Pesquisa Nacional de Saúde 2013. Rio de Janeiro: IBGE; 2014.

17. Duca GFD, Silva MCD, Hallal PC. Incapacidade funcional para atividades básicas e instrumentais da vida diária em idosos. Rev Saude Publica 2009; 43(5):796805.

18. Silva ITD, Pinto Junior EP, Vilela ABA. Autopercepção de saúde de idosos que vivem em estado de corresidência. Rev Bras Geriatr e Gerontol 2014; 17(2):275-287.

19. Cabena P, Hadjinian P, Stadler R, Verhees J, Zanasi A. Discovering Data Mining: From Concept to Implementation. Upper Saddle River: Prentice Hall; 1998.

20. Vianna RCXF, Moro CMCDB, Moysés SJ, Carvalho D, Nievola JC. Mineração de dados e características da mortalidade infantil. Cad Saude Publica 2010; 26(3):535-542.

21. Kleinbaum DG, Klein M. Logistic Regression. New York: Springer New York; 2010.

22. Carmo JFD, Oliveira ERA, Morelato RL. Incapacidade funcional e fatores associados em idosos após o Acidente Vascular Cerebral em Vitória - ES , Brasil. Rev Bras Geriatr e Gerontol 2016; 19(5):809-818.

23. World Health Organization (WHO). International Classification of functioning, disability and health: ICF. Geneva: WHO; 2001.

24. Brasil. Ministério da Saúde (MS). Portaria ${ }^{\circ} 2.528$, de 19 de Outubro de 2006. Aprova a Política Nacional de Saúde da Pessoa Idosa. Diário Oficial da União 2006; 19 out.

25. Areosa SVC, Benitez LB, Wichmann FMA. Relações familiares e o convivívio social entre idosos. Textos Contex 2012; 2(1):184-192.

26. Gomide MFS, Pinto IC, Figueiredo LAD. Acessibilidade e demanda em uma unidade de pronto atendimento: Perspectiva do usuário. Acta Paul Enferm 2012; 25(2):19-25.

27. Jerez-Roig J, Souza DLB, Andrade FLJPD, Lima Filho BF, Medeiros RJ, Oliveira NPDD, Cabral Neto SM, Lima KC. Autopercepção da saúde em idosos institucionalizados. Cien Saude Colet 2016; 21(11):33673375.
28. Belém PLDO, Melo RLPD, Pedraza DF, Menezes TND. Autoavaliação do estado de saúde e fatores associados em idosos cadastrados na Estratégia Saúde da Família de Campina Grande, Paraíba. Rev Bras Geriatr e Gerontol 2016; 19(2):265-276.

29. Cesari M, Prince M, Thiyagarajan J, De Carvalho I, Bernabei R, Chan P, Gutierrez-Robledo L, Michel J, Morley J, Ong P, Rodriguez Manas L, Sinclair A, Won C, Beard J, Vellas B. Frailty: An Emerging Public Health Priority. J Am Med Dir Assoc 2016; 17(3):188192.

30. Gong CH, Kendig H, He X. Factors predicting health services use among older people in China: An analysis of the China Health and Retirement Longitudinal Study 2013. BMC Health Serv Res 2016; 16(63):1-16.

31. Malta DC, Bernal RTI, Lima MG, Araújo SSC, Silva MMAD, Freitas MIDF, Barros MBDA. Doenças crônicas não transmissíveis e a utilização de serviços de saúde: análise da Pesquisa Nacional de Saúde no Brasil. Rev Saude Publica 2017; 51(1):1-10.

32. Caldas CP, Veras RP, Motta LBD, Guerra ACLC, Carlos MDJ, Trocado CVM. Atendimento de emergência e suas interfaces: o cuidado de curta duração a idosos. J Bras Econ da Saúde 2015; 7(1):62-69.

33. Lopes MCBT, Lage JSS, Vancini-Campanharo CR, Okuno MFP, Batista REA. Factors associated with functional impairment of elderly patients in the emergency departments. Einstein 2015; 13(2):209-214.

34. Calero-García MJ, Ortega AR, Navarro E, Calero MD. Relationship between hospitalization and functional and cognitive impairment in hospitalized older adults patients. Aging Ment Health 2017; 21(11):1164-1170.

35. Martínez Velilla N, Casas Herrero A, Zambom Ferraresi F, Suárez N, Alonso Renedo J, Contín KC, Asteasu MLSD, Echeverria NF, Lázaro MG, Izquierdo M. Functional and cognitive impairment prevention through early physical activity for geriatric hospitalized patients: study protocol for a randomized controlled trial. BMC Geriatr 2015; 15(1):1-9.

36. Zaslavsky O, Zisberg A, Shadmi E. Impact of functional change before and during hospitalization on functional recovery 1 month following hospitalization. $J$ Gerontol A Biol Sci Med Sci 2015; 70(3):381-386.

37. Tamai SAB, Paschoal SMP, Litvoc J, Machado AN, Curiati PK, Prada LF, Jacob Filho W. Impacto de um programa de promoção da saúde na qualidade de vida do idoso. Einstein 2011; 9(1):8-13.

38. Ferreira DN, Matos DL, Loyola Filho AIDL. Absence of routine medical consultation among hypertensive and/or diabetic elders: an epidemiological study based on the Brazilian National Household Survey 2008. Rev Bras Epidemiol 2015; 18(3):578-594.

Artigo apresentado em 11/05/2018

Aprovado em 31/07/2018

Versão final apresentada em 02/08/2018 